\title{
Reduction of needlestick injuries in obstetrics and gynecology trainees and attendings through introduction of blunt needles on labor and delivery unit
}

\author{
Caroline Foust-Wright ${ }^{*}$, K Lauren Barnes ${ }^{2}$, Angel Johnson ${ }^{3}$ and Samantha Pulliam ${ }^{4}$ \\ ${ }^{1}$ Maine Medical Partners, Women's Health Urogynecology, USA \\ ${ }^{2}$ Division of Female Pelvic Medicine \& Reconstructive Surgery, University of New Mexico, USA \\ ${ }^{3}$ Department of Urogynecology, Greater Boston Urology, USA \\ ${ }^{4}$ Division of female pelvic medicine \& reconstructive surgery, University of North Carolina, USA
}

\section{Introduction}

\section{Incidence}

There are an estimated 385,000 accidental needlestick injuries (NSI) of healthcare workers annually in the United States [1]. 16,000 healthcare workers are exposed to HIV through needlestick injuries annually, with even more having exposure to hepatitis B and C [1]. The seroconversion rate for HIV after a single percutaneous exposure has been estimated by the CDC to be approximately $0.3 \%$, hepatitis $\mathrm{B}$ 6-30\%, and hepatitis C 1.8\% [1].

\section{Surgical trainee-specific risk}

Surgeons in training of all types have the greatest risk of exposure due to the high volume of procedures performed while in training and the increased propensity for injury while learning new technical skills and working long hours [2]. A study published in JAMA in 1992 evaluating percutaneous injuries during surgical procedures confirmed that the rate of NSI for resident surgeons was the highest out of all personnel in the OR, at $2.7 \%$ risk per procedure [3]. Vaginal surgery was considered to be the highest risk, with up to $21 \%$ of cases having a NSI occur [3]. Nearly half of these needlestick exposures during vaginal surgery were further complicated by recontact with the patient, thus providing a dual-exposure of the provider to the patient and the patient to the provider, compromising patient safety [3].

A study published in JAMA in 2006 looked specifically at interns and their risk of percutaneous injuries and found the mean rate per intern-month of percutaneous injuries to be approximately 0.03 [4]. General surgery had a significantly higher rate at 0.07 per internmonth, with only OBGYN interns at higher risk at nearly 0.1 per intern-month [4].

For non-vaginal procedures, closure of the abdominal fascia carries the highest risk component of percutaneous injury with a suture needle [3]. Glove perforation is often used as proxy in assessing the efficacy of techniques to reduce percutaneous injuries. In A study by Arena, nearly one quarter of Cesareans and half of perineal laceration repairs had glove perforations identified by procedure end [5]. Resident trainees have the added risk of learning new technical skills while performing these high risk procedures as well as the effect of fatigue and night shifts.

\section{Fatigue-associated risk}

Medical trainees account for 33\% of reported sharps-related injuries each year, though account for much less than one-third of healthcare workers [3]. The majority of exposures by trainees are not reported, thus the true incidence is probably higher than we suspect [2]. Though there are several likely reasons trainees are at higher risk, fatigue is certainly one risk factor. A study by Fisman evaluating the effect of fatigue on risk of a sharps-related injury was conducted at 5 academic medical centers [6]. This case-crossover study included all employees who reported a sharps-injury over a 4 year period and looked at hours worked in the past week, worked on the day of injury, and a self-assessment of fatigue at time of injury. Trainees worked significantly more hours per week and slept less the night before an injury than did other healthcare workers, with a higher fatigue level [6]. In this study, fatigue increased injury risk in the trainee population 3 -fold [6].

University of Texas Medical School in Houston conducted a study to determine if there was a day-night pattern in accidental exposures to blood-borne pathogens among trainees. This study analyzed a 5-year period of all reported exposures [7]. There were 40 exposures per hour per 1000 doctors in training during the day, with 60 exposures per 1000 trainee hours at night, with a $50 \%$ higher risk of sustaining a bloodborne pathogen exposure when working nights than when working days [7]. The 2006 JAMA study also found a 2 times greater likelihood of percutaneous injury in night shift than day shift [4].

\section{Blunt suture needles}

Blunt suture needles have been available since the early 1990s. These are curved suture needles that have a relatively blunt tip. They were introduced due to the decreased likelihood of causing percutaneous injuries as they do not penetrate skin as easily and require more pressure to penetrate tissue.

A study was conducted at 3 New York City teaching hospitals in collaboration with the CDC shortly after blunt needle introduction to

Correspondence to: Caroline Foust-Wright, MD, MBA, Women's Health Urogynecology, 100 Brickhill, Suite 203, South Portland, ME, USA, E-mail: cfoustwrig@mmc.org

Received: July 22, 2017; Accepted: August 12, 2017; Published: August 15, 2017 
review their safety in gynecologic surgery. Both conventional and blunt needles were available for use, with blunt needles used in more than a quarter of procedures. There were no percutaneous injuries during the study period involving blunt needles [8]. The results revealed an $87 \%$ relative risk reduction of NSI in gynecologic surgery if $50 \%$ of suture needles used in a procedure are blunt tip [8]. Only $6 \%$ of surgeons reported technical difficulties with blunt needles, including difficulty penetrating tissue and needle slippage, but no clinically important differences were found [8].

A randomized controlled trial published in Obstetrics \& Gynecology in 2009 evaluated the risk reduction of needlestick injuries during Cesarean deliveries [9]. Glove perforation was the primary outcome, as it is an accepted proxy for needlestick injuries. The number needed to treat to avoid one glove perforation was only 9.7 [9]. When considering the number of Cesareans performed by obstetric trainees, this is a significant potential risk reduction.
Based upon the evidence supporting the increased safety of blunt needles for reduction of injuries, in 2005 the American College of Surgeons issued a statement supporting universal adoption of blunt needles for all fascial closures and encouraged use of blunt needles in all other appropriate surgical applications [10]. ACOG has issued no similar statement at this time. A Cochrane review in 2011 noted that there is high quality evidence that the use of blunt needles appreciably reduces the risk of exposure to blood and bodily fluids for surgeons and their assistants over a range of operations. It is unlikely that future research will change this conclusion [12].

\section{Methods}

The PDSA cycle was conducted as detailed in Figure 1. Prior to initial OBGYN Grand Rounds presentation, the residents were queried about the number of needlestick injuries they experienced in the prior year. This survey included questions about total number experienced,

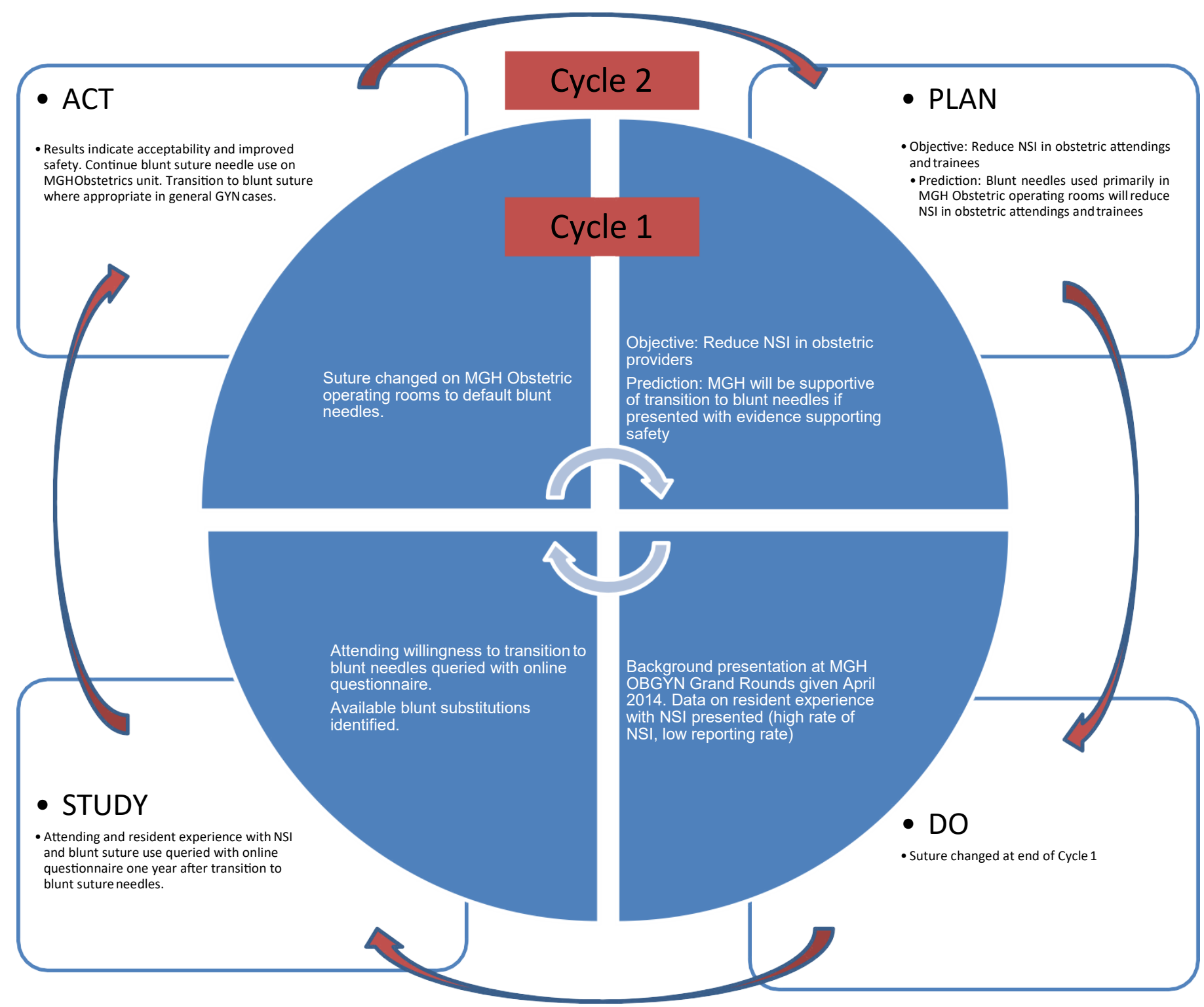

Figure 1. This figure demonstrates the PDSA (Plan, Do, Study, Act) cycles that were utilized during the quality project to reduce needlestick injuries (NSI) in obstetric providers. This study was conducted at a large academic institution $(\mathrm{MGH})$ with residents and medical studies participating in procedures. 
number that were not reported, and reasons why these occupational exposures were not reported. This information was presented at the Grand Rounds presentation, prompting discussion about the importance of trainee safety and the need for transition to blunt needles as a safer option.

All attending physicians with obstetric privileges at a single large academic center were queried regarding their perceptions of blunt needles and their personal experiences with needlestick injuries. Following this survey, suture needles in the hospital Cesarean theaters were changed to blunt needles, regardless of physician preference.

A one-year follow-up survey was sent to both the residents and the attendings regarding their personal experiences with NSI in the one year that blunt needles were in use. A Likert-style scale was used to assess their preference for use of blunt needles. The perception of the impact of blunt needles on occupational exposure safety was queried in the attendings. All surveys were sent via electronic mail, with a survey link allowing anonymous response. The survey link was only sent once, with no follow-up reminders.

\section{Results}

Of the 35 attending providers with obstetric privileges at this large tertiary academic center, $74 \%$ participated in the pre-change survey and $40 \%$ participated in the post-change survey. Prior to the introduction of blunt needles, $28 \%$ of attendings had at least one NSI in the prior year. Following the introduction of blunt suture needles, only 1 NSI occurred in the responding attendings, with $93 \%$ reporting no NSI. None of the needlestick injuries sustained by the attending physicians in either query were reported to occupational health (total of 9 needlesticks went unreported by attendings). There were 8 needlesticks sustained by responding attendings in the year preceding the transition to blunt needles versus 1 in the year following the transition to blunt needles ( $p=0.05$ ) (Figure 2). Attending perception of blunt needle does not reflect this finding. Prior to the introduction, greater than $70 \%$ of attendings believed that blunt needles would reduce NSI in residents and attendings; following the introduction of NSI on the obstetrics ward, only $29 \%$ still felt this to be true, despite the actual reduction in NSI occurrence among attendings with $\mathrm{p}<0.01$ for pre/post change (Figure 2).

Of the 44 OBGYN residents, $41 \%$ responded to the pre-change survey and $27 \%$ responded to the post-change survey. Of the responding residents, $61 \%$ had sustained at least 1 NSI in the preceding year in the initial query; $50 \%$ sustained at least 1 NSI following the introduction to blunt suture needles. None of these needlestick injuries actually occurred with blunt suture needles. Of the 17 total NSI recalled by responding residents in the initial query, only $41 \%$ were reported to occupational health. At the 1-year follow-up survey, a total of 10 NSI were recalled, with a reporting rate of $50 \%$ (Figure 2).

Greater than $40 \%$ of residents and attendings use blunt suture needles and find them acceptable. Only $14 \%$ of attendings and $17 \%$ of residents reported that they only use blunt suture needles when they have no other choice.

\section{Discussion}

Needlestick injuries are incredibly common. As a study by Makary noted in 2007 in the New England Journal of Medicine, 99\% of surgical residents have at least one NSI while in training, with a mean of greater

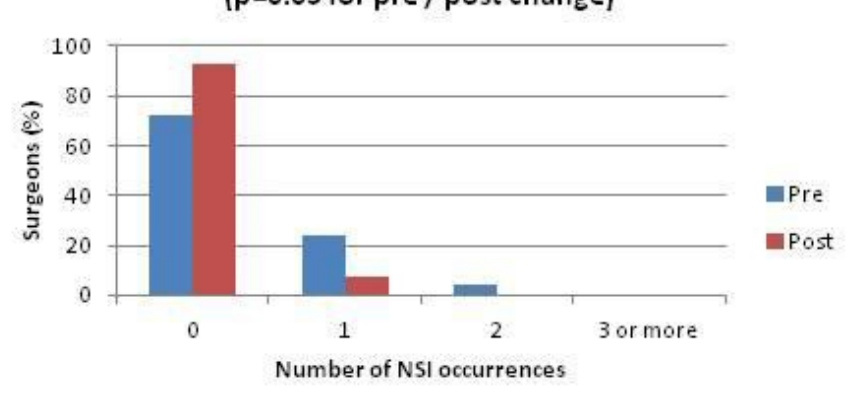

Non-reported attending NSI in preceding

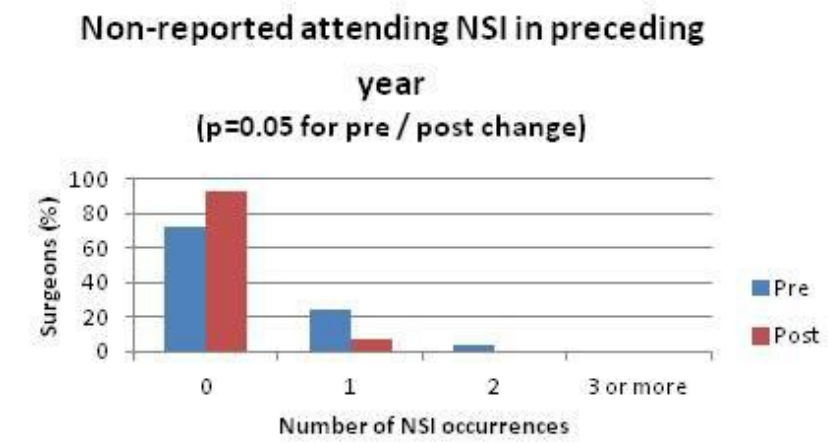

\section{Attending NSI Occurrence in preceding year (reported and non-reported) ( $\mathrm{p}=0.05$ for $\mathrm{pre} /$ post change)}

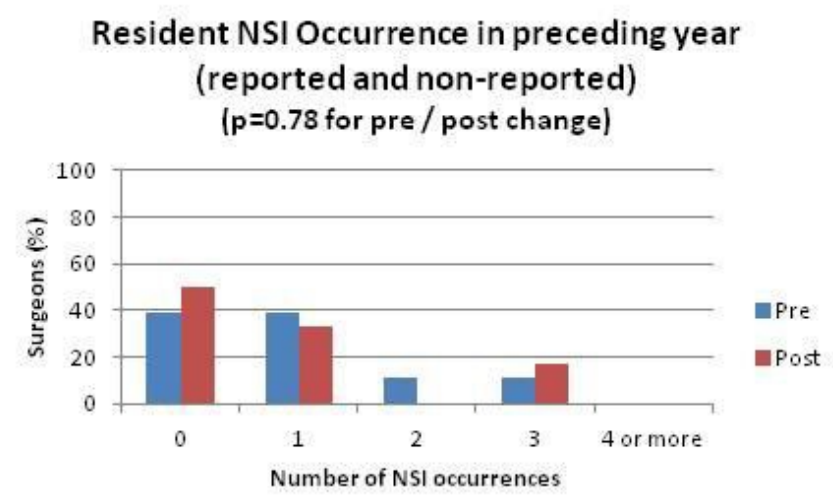

Non-reported resident NSI in preceding year ( $p=0.69$ for $\mathrm{pre} /$ post change)

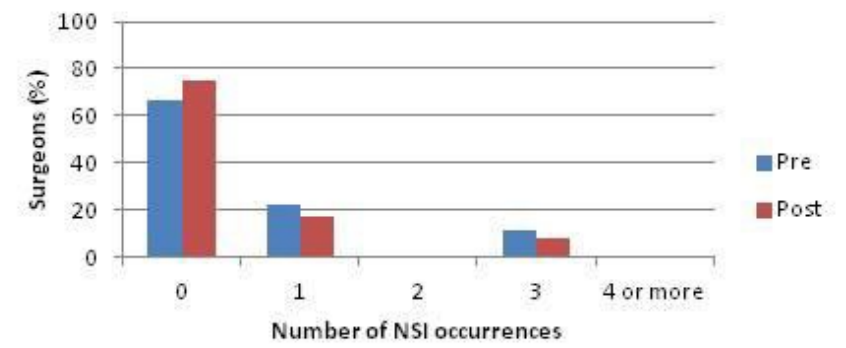

Figure 2. This figure demonstrates the change in needlestick injuries (NSI) in attending physicians from prior to the PDSA cycle and following completion of the interventions. The upper two graphs demonstrate all NSI, reported and unreported, and the lower two graphs demonstrate the non-reported NSI occurrences. Of note, the incidence of unreported NSI in attending obstetricians was significantly reduced following the interventions of the PDSA cycle. 
than 5 needlestick injuries by the fourth year of training [12]. Our data demonstrated a 50-61\% incident of resident NSI per year. Makary also found that more than half of the most recent injuries sustained were not reported to employee health, with lack of time was reported as the most frequent reason for not seeking care for a sustained needlestick injury, which was also reproduced in our study [12].

Through increased use of blunt needles there was a significant decrease NSI in attending physicians and a notable decrease in trainees, though not statistically significant. This lack of statistical significance among the resident responders may be due to the low participation rate and the high NSI occurrence. Though the attending physicians did not tend to believe that the blunt needles improved the incident of NSI, this was demonstrated in both attending physicians and residents. One attending obstetrician stated "Honestly I thought the blunt needles were a great idea... but in practice I feel like it takes more force to get through the fascia in particular and worry that it might cause learners to actually stick themselves more."

The increased rate of reporting NSI was an unexpected and interesting finding generated out of this study likely caused by improvement in departmental education. The reasons that needlestick injuries went unreported are varied. Attending physicians responses noted that the "patient was low risk and known to be HIV and Hepatitis B neg. I would not take AZT, didn't want to jump the hoops again- have done so in the past", "low risk stick", and "not helpful for me to report it". Residents felt that the reporting process was "too time consuming", "too much hassle, couldn't leave work or couldn't leave the case", or "we were very busy on L\&D and I was the chief in charge. I could not afford the time to go to occupational health. I checked pt labs." Increasing education about NSI will likely improve reporting of these incidents and lead to better care of trainees and decreasing burden of reporting paperwork requirements in line with Occupational Safety \& Health Administration (OSHA) requirements may improve overall reporting [13].

\section{Conclusion}

The introduction of blunt suture needles on the labor unit at an academic center resulted in a decrease in total recalled needlestick injuries sustained by residents and attendings. The decrease was most notable among the attending physicians, despite their perception that blunt needles were not safer.
Further education on the necessity of reporting, setting a positive and professional example for trainees, and further efforts for reduction of occupational exposures is needed. In this era of increased occupational hazard from exposures to blood borne pathogens, transition to safer devices where possible is a necessity. Blunt needles are an acceptable alternative to the sharp suture needles and thus should be utilized wherever feasible.

\section{References}

1. Center for Disease Control and The National Institute for Occupational Safety and Health (NIOSH) Stop Sticks Campaign. http://www.cdc.gov/niosh/stopsticks/ sharpsinjuries.html Accessed 6/19/2016

2. Brasel KJ, Mol C, Kolker A, Weigelt JA (2007) Needlesticks and surgical residents: who is most at risk? J Surg Educ 64: 395-398. [Crossref]

3. Tokars JI, Bell DM, Culver DH, Marcus R, Mendelson MH, et al. (1992) Percutaneous injuries during surgical procedures. JAMA 267: 2899-2904. [Crossref]

4. Ayas NT, Barger LK, Cade BE, Hashimoto DM, Rosner B, et al. (2006) Extended work duration and the risk of self-reported percutaneous injuries in interns. JAMA 296: 1055-1062. [Crossref]

5. Arena B, Maffulli N, Vocaturo I, Scognamiglio G (1991) Incidence of glove perforation during caesarean section. Ann Chir Gynaecol 80: 377-380. [Crossref]

6. Fisman DN, Harris AD, Rubin M, Sorock GS, Mittleman MA (2007) Fatigue increases the risk of injury from sharp devices in medical trainees: results from a case-crossover study. Infect Control Hosp Epidemiol 28: 10-17. [Crossref]

7. Parks DK, Yetman RJ, McNeese MC, Burau K, Smolensky MH (2000) Day-nigh pattern in accidental exposures to blood-borne pathogens among medical students and residents. Chronobiol Int 17: 61-70. [Crossref]

8. Mendelson M et al. (1997) Evaluation of Blunt Suture Needles in Preventing Percutaneous Injuries Among Health-Care Workers During Gynecologic Surgical Procedures. MMWR Morb Mortal Wkly Rep 46: 25-29. 7. [Crossref]

9. Sullivan S, Williamson B, Wilson LK, Korte JE, Soper D (2009) Blunt Needles for Reduction of Needlestick Injuries During Cesarean Delivery. Obstet Gynecol 114: 211216. [Crossref]

10. Bulletin of American College of Surgeons (2005). 90: 11.

11. Saarto A, Verbeek J, Lavoie M, Pahwa M (2011) Blunt versus sharp suture needles for preventing percutaneous exposure incidents in surgical staff. Cochrane Database Syst Rev. [Crossref]

12. Makary MA, Al-Attar A, Holzmueller CG, Sexton JB, Syin D, et al. (2007) Needlestick injuries among surgeons in training. N Engl J Med 356: 2693-2699. [Crossref]

13. Occupational Exposure to Bloodborne Pathogens; Needlestick and other sharps injuries; final rule. 66 Federal Register 12 (2001). p 5318-25.

Copyright: $(02017$ Foust-Wright C. This is an open-access article distributed under the terms of the Creative Commons Attribution License, which permits unrestricted use, distribution, and reproduction in any medium, provided the original author and source are credited. 